\title{
Automated Multimodal image fusion for brain tumor detection
}

\author{
Harpreet Kaur ${ }^{1}$, Deepika Koundal², Virendar Kadyan ${ }^{3}$, Navneet Kaur ${ }^{4}$, and Kemal Polat ${ }^{5, *}$ \\ ${ }^{1}$ Chitkara University Institute of Engineering and Technology, Chitkara University, India. Email: \\ harpreet.kaur.045@gmail.com \\ ${ }^{2}$ Department of Virtualization, School of Computer Science, University of Petroleum and Energy Studies, Dehradun, \\ India. Email: dkoundal@ddn.upes.ac.in \\ ${ }^{3}$ Department of Informatics, School of Computer Science, University of Petroleum and Energy Studies, Dehradun, India. \\ Email: vkadyan@ddn.upes.ac.in \\ ${ }^{4}$ Department of Computer Science, Baba Banda Singh Bahadur Engineering College, Fatehgarh Sahib, Punjab, India. \\ Email: navneet.sehgal@bbsbec.ac.in \\ ${ }^{5}$ Department of Electrical and Electronics Engineering, Faculty of Engineering, Bolu Abant Izzet Baysal University, \\ 14280, Bolu, Turkey. Email: kpolat@ibu.edu.tr \\ * Corresponding Author
}

How to cite this paper: Harpreet Kaur, Deepika Koundal, Virendar Kadyan, Navneet Kaur, and Kemal Polat (2021). Automated Multimodal image fusion for brain tumor detection. Journal of Artificial Intelligence and Systems, 3, 68-82. https://doi.org/10.33969/AIS.2021.31005

Received: May 17, 2021

Accepted: May 31, 2021

Published: June 3, 2021

Copyright (C) 2021 by author(s) and Institute of Electronics and Computer. This work is licensed under the Creative Commons Attribution International License (CC BY 4.0).

http://creativecommons.org/licenses/by/4.0/

(c) (i) Open Access

\begin{abstract}
In medical domain, various multimodalities such as Computer tomography (CT) and Magnetic resonance imaging (MRI) are integrated into a resultant fused image. Image fusion (IF) is a method by which vital information can be preserved by extracting all important information from the multiple images into the resultant fused image. The analytical and visual image quality can be enhanced by the integration of different images. In this paper, a new algorithm has been proposed on the basis of guided filter with new fusion rule for the fusion of different imaging modalities such as MRI and Fluorodeoxyglucose images of brain for the detection of tumor. The performance of the proposed method has been evaluated and compared with state-of-the-art image fusion techniques using various qualitative as well as quantitative evaluation metrics. From the results, it has been observed that more information has achieved on edges and content visibility is also high as compared to the other techniques which makes it more suitable for real applications. The experimental results are evaluated on the basis of withreference and without-references metric such as standard deviation, entropy, peak signal to noise ratio, mutual information etc.
\end{abstract}

\section{Keywords}

Medical, Brain, Magnetic resonance imaging, Fluorodeoxyglucose, Principal Component Analysis, Multi resolution singular value decomposition 


\section{Introduction}

Image fusion (IF) is widely used in medical domain for determining the better visibility of diagnosis. Several researchers have worked on different type of imaging modalities such as Magnetic Resonance Imaging (MRI), Computer Tomography (CT), and Fluorodeoxyglucose (FDG) images for the detection of tumor. Multimodal image fusion is very helpful in capturing the different types of information from diverse body parts. IF is a process in which two or more multi modalities are integrated into the fused image. Thus, Medical IF is helpful for features extraction from diverse modalities that are not normally visible in the single image.

As per literature [15-20], many IF frameworks have been developed for the fusion of diverse images in medical domain. It is generally categorized as pixel, feature and decision level [1]. Pixel level image fusion is performed at the lowest level which is the simplest of all fusion methods. It has integrated the intensity values of two source images for computing the average to produce a resultant fused image [2]. There are certain drawbacks of pixel level fusion such as it produced the blurring effect while smoothening the sharper edges of the resultant fused image. Another category of image fusion is the feature level image fusion which is at the middle level amongst the IF levels. In this, image features such as textures and edges are taken out initially from the source images. Subsequently, fusion method based on these features performed well to match the certain selection criteria [3]. Decision level IF techniques are used to merge information at advanced level of abstraction. For the purpose of extracting information from source images, the source images are processed individually [3]. It is classified on the basis of datasets such as multi-temporal, multifocus, multi-scale, multi-spectral and multi-sensor. It has been widely used in many different applications such as medical diagnosis, surveillance, photography and remote sensing. Hence, in this paper, the proposed image fusion method is based on pixel level IF. The well-known pixel level frameworks are based on guided filter (GF), multi resolution singular value decomposition (MSVD) and principal component analysis (PCA).

As per the literature, the guided image filter has been presented for the fusion of MRI and CT images by Bavirisetti et al. [4]. In this, the silent information from the images has been extracted (GF) and the best edge preserving information has been given by the guided filter. In another work, the image fusion with guided filter has been introduced by Li S. et al. [5] in which GF works as a boundary smoothing and preserving operator similar to the admired bilateral filter. It has enhanced the performance of the boundaries. It has a hypothetical link with Laplacian matrix. It is a fast and non-estimated linear time algorithm, whose density is not dependent on the mask size. This filter is more efficient and effective in graphics and computer vision 
applications with joint up-sampling, haze removal, detail smoothing and noise reduction. Further, Multi resolution singular value decomposition (MSVD) fusion method has been introduced by Naidu et al. [6]. Wavelet transformation (WT) and MSVD resembled to each other. In this, signals have been decomposed into low pass finite impulse filter and high pass finite impulse filter. At each decomposition level, the filter's output has been decreased and decomposed into the next level and this process has been reiterated for achieving the next successive levels. Furthermore, the MSVD, WT and Laplacian pyramid techniques are compared by Sireesha et al. [7]. From the experimental results, it has been observed that MSVD has achieved better performance in comparison to the WT in terms of Root Mean Square Error (RMSE) value, which is low and Peak Signal to Noise Ratio (PSNR) value, which is high. Further, the pixel level IF method using Principal Component Analysis (PCA) and WT has been introduced by Naidu et al. [8]. PCA is a statistical method based on orthogonal transformation for the conversion of set of observations of possibly correlated variable into the principal and also used as a dimension reduction technique. In this, PCA is used as a statistical tool for transforming the correlated variables into the uncorrelated variables and has been found better than WT. The main drawback of PCA is spectral degradation and color distortion. Bai L. et al. has reported the review of various multi-spectral image fusion methods [9]. Whereas Rana et al. presented a comparative analysis of various medical IF techniques [10]. Nobariyan B. K. et al. [11] had proposed a new medical image fusion paradigm by fusing the high-resolution anatomic image with functional images for the enhancement of spatial resolution. When came to it, after the registration procedure, perform Green (Y), Blue (Cb), Red ( $\mathrm{Cr}$ ) (YCbCr) on the multispectral picture, red difference chromatic element and blue difference chromatic element and afterward DWT picture image fusion calculation dependent on pulse-coupled neural network (PCNN) was connected to merge the MRI picture and the luminance segment. In the past years, several researchers did lot of work in the area of image fusion of multimodal image using diverse techniques but still some issues such as unclear visibility of edges, blurred edges are remain unaddressed. Hence, a new multimodal image fusion technique is needed to be developed that can address the issues faced by the existing state-of-art techniques.

Though, various image fusion methods have been introduced for the fusion of multimodal medical image. But still most of them has focused on fusing colored images or grayscale images only. This paper proposes a novel method for the fusion of grayscale as well as colored multi-modal medical images. In this paper, image fusion has been done with the MRI and Fluorodeoxyglucose (FDG) images. MRI is a graycale image and FDG is a colored image. MRI is used to capture the soft tissue 
Harpreet Kaur et al.

structures like heart, eyes and brain whereas FDG imaging is utilized for the accurate detection and diagnosis of malignant tissues such as recurrent colorectal cancer, lymphoma and lung cancer. However, it is provide complementary information in one image from multiple images for more content visibility and it's also useful for patient treatments. Moreover, proposed technique has been compared with the exiting multi modal image fusion techniques. The contributions of this paper are as follow: (i) An image fusion technique has been proposed for fusing the multimodal images which is based on the Guided filter with the new fusion rule. (ii) The performance of proposed technique has been evaluated on the basis of qualitative as well as quantitative evaluation metrics. (iii) It provided more information of edges as well as its content visibility is also high as compared to other techniques. (iv) It is also helpful for extracting the features from different modalities that are not normally visible in the single image.

Rest of the paper is organized as follow: Materials and methods are highlighted in Section II whereas Section III discussed the experimental results. Section IV summarizes the conclusion.

\section{Material and Methods}

\subsection{Dataset}

For experimental results, the image datasets of MRI and FDG images are used, which is publically available. Image datasets are collected from “http://www.med.harvard.edu/AANLIB/home.html”. The MRI images and FDG images are available in gray and color format respectively. It includes normal brain images, cerebrovascular disease, neoplastic disease and degenerative disease images. Here, results have been taken on images of brain tissue. In this work, fusion has been done with gray and color images. These results have been tested on 20 images from the dataset. In this experimental work, implementation has been done using Matlab 2017 and AMD A4 processor with 2GB memory.

\subsection{Methods}

\subsubsection{Guided filter}

Guided filter is used as smoothing as well as edge preserving filter. It has the capability of preserving the edge information as well as of smoothening the source image. It computes the output pixels by taking the neighborhood information around the pixel of an image. It is widely used in image enhancement, image matting and preservation of images. The present IF process applies the GF for the enhancement of the quality of the fused images. It has the feature of preserving the smoothness of the images and did not get affected by various gradient reversal artifacts like other 
filters [4]. It has defined the linear translation-variant filtering process that involved an input image $I$, supervised image $S$, and an output image $O$. Both $S$ and $I$ are given beforehand and can be same. The filtering output is expressed as a weighted average:

$$
O_{l}=\sum_{j} W_{i j}(S) I_{j},
$$

where $i$ and $j$ are pixel levels. The window $W_{i j}$ is a function of the supervised image $S$ and independent of $I$.

\subsubsection{Proposed technique}

There are considering two cases. In the first case MRI is used as an input image $I(i, j)$ and FDG is used as a supervised image. $S(i, j)$. In the second case MRI is used as a supervised image and FDG is used as an input image. The framework of the proposed image fusion technique is illustrated in Figure 1. Following are the major steps that are implemented for proposed technique are as follow:

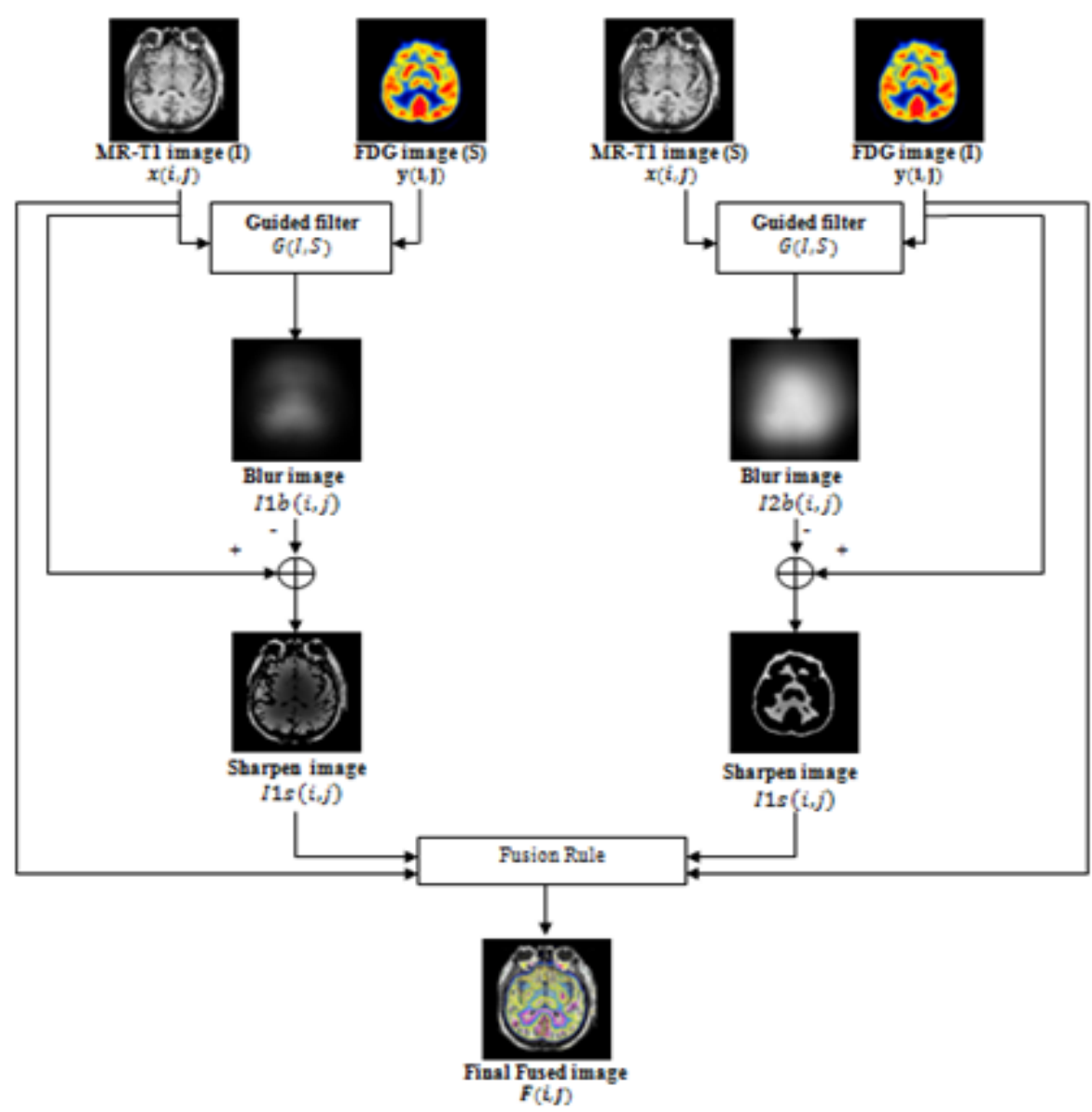

Figure 1. Framework of proposed image fusion technique. 
Firstly, guided filter $G(I, S)$ has been applied on the input image (I) and supervised images (S), respectively in order to get the blur image $G_{b}$. Guided filter has been applied using equation

$$
G_{b}=G(I, S)
$$

Subsequently, subtraction operation has been applied on blur image $G_{b}$ and input image $I_{s}$.

$$
I_{s}=I-G_{b}
$$

Then, proposed fusion rule has been applied on $I_{s}$ to obtain the fused image $F(i, j)$. The algorithm of the proposed technique is as follows:

Input: Two input images Magnetic Resonance Imaging (MRI) $(I 1(i, j))$ and Fluorodeoxyglucose (FDG) $(I 2(i, j))$. MRI is a gray image and FDG is a color image.

Output: Fused image $F(i, j)$.

Initialize parameters: radius ' $r$ ' $=25$, epsilon 'eps'=1.5 and window 'con_wsize'=3.

Step 1. There are considering two cases. In the first case MRI is used as an input image (I1) and FDG is used as a supervised image $(S)$. In the second case MRI is used as a supervised image and FDG is used as an input image.

Step 2. For $i=1$ to 3,

$$
\begin{array}{r}
I(:,:, 1)=I 1 / 255 ; \\
I(:,:, 2)=I 2(:,:, i) ;
\end{array}
$$

Step 2a. Guided filter has been applied on using equation 1 on both the cases and get blurred images $(I 1 b(i, j)$ and $I 2 b(i, j))$ using equation 2. Here, $(I 1 b(i, j)$ and $I 2 b(i, j))$ is represent the blur image.

Step 2b. Subtract the blurred image from the input image and get sharpen image $((I 1 s(i, j))$ and $(I 2 s(i, j)))$ in the both cases using equation 3. Here, $((I 1 s(i, j))$ and $(I 2 s(i, j)))$ is represent the subtracting image.

Step 3. New proposed fusion rule has been applied on the both images content. Go to Step 2.

$F(i, j)=(I 1(i, j) * w x(i, j)+I 2(i, j) * w y(i, j)) /(w x(i, j)+w y(i, j))$

(4)

Where, $I 1(i, j)$ and $I 2(i, j)$ are input image. $w x(i, j)$ and $w y(i, j)$ are the weight variables of the images.

Step 4. End of For loop.

Step 5. In the last step, the final fused image $(F(i, j))$ with preserved edges is obtained.

The new proposed fusion rule is discussed as below:

In the first step evaluate the corresponding pixel to each and every pixel in the input images based on the horizontal and vertical strength of the edges. Then, find the weight value of a pixel at the location $(x, y)$ in an image from a square window $w$ of size $n^{*} n$ with respect to the adjacent pixels in the image. Subsequently, a matrix and 
determine its covariance matrix on the basis of rows and column variables by using following equation:

$$
\operatorname{cov}(Y)=E\left[(Y-E[Y])\left(Y-E[Y]^{T}\right]\right.
$$

Then, calculate the unbiased estimate $C_{H}^{x, y}(Y)$ of a covariance matrix for each pixel location $(x, y)$ as

$$
C_{H}^{x, y}(Y)=\frac{1}{m-1} \sum_{j=1}^{n}\left(Y_{j}-\bar{Y}\right)\left(Y_{j}-\bar{Y}\right)^{T}
$$

where $Y_{j}$ signifies the $j_{\text {th }}$ observation of the $m$ dimensional variable and $\bar{Y}$ signifies the average observation, $C_{H}{ }^{x, y}(Y)$ denotes the variance vector.

After this, evaluation of Eigen values $\delta_{H}^{j}$ of $C_{H}^{x, y}(Y)$ has been performed. The number of Eigen values is $n$ according to the matrix size $n * n$. Subsequently, all these Eigen values have been computed to find out the strength of horizontal edge $\alpha_{H}$.

$$
\alpha_{H}(x, y)=\sum_{j=1}^{n} \delta_{H}^{j}
$$

Comparably, the strength of each vertical edge has been considered. Each row has been considered as a variable and column as an observation for performing the assessment of unbiased estimate $C_{V}^{x, y}$, and for computing the Eigen values $\delta_{V}^{j}$ of $C_{V}^{x, y}$. Finally, final vertical edge strength has been attained by adding these eigen values $\alpha_{V}$ as,

$$
\alpha_{V}(x, y)=\sum_{j=1}^{n} \delta_{V}^{j}
$$

In this step, the weight value $W(x, y)$ of each and every pixel corresponding to the location $(x, y)$, and consider the sum of $\alpha_{H}(x, y)$ and $\alpha_{V}(x, y)$.

$$
W(x, y)=\alpha_{H}(x, y)+\alpha_{V}(x, y)
$$

The above step is repeated for corresponding to each and every pixel comprised in an image for assigning the weight values. Where, the weight value of the pixels relies on the strength of the edges instead of the intensity values of the pixels.

\section{Results and Discussion}

\subsection{Fusion evaluation metrics}

For result analysis, different types of evaluation metrics have been used for several multimodal IF techniques. For quantitative analysis of several multimodal IF techniques, both "with reference" and "without reference" evaluation metrics have been computed.

There are two types of evaluation metrics. Without references and with references evaluation metrics are discussed below: 


\subsubsection{Without references evaluation metric}

\section{1) Standard Deviation}

SD is reflecting the clarity and contrast of gray images $[12,13]$. The value of SD can be computed using equation (1) represented below:

$$
\mathrm{SD}=\sqrt{\frac{1}{P \times Q} \sum_{i=1}^{P} \sum_{j=1}^{Q}\left(F_{i j}-M E A N\right)^{2}}
$$

If SD value is high, it means it contains more noise in the resultant image. Here, $P$ and $Q$ are the pixel values of images. $F_{i j}$ is a fused image.

2) Entropy

EN is employed for the evaluation of the image information that is sensitive to noise. It is used to provide the huge content information from the image [12,13] which is defined as:

$$
\sum_{l=0}^{L-1} p_{l} \log _{2} p_{l}
$$

where, $L$ is used to represent the gray levels count and $p_{l}$ is used to determine the histogram of equivalent gray levels. The higher entropy value means better performance and more information obtained in the image fusion.

3) Mean ( $\mu)$

Mean evaluation metric is used to determine the contrast for image fusion.

$$
\bar{F}=\mu=\frac{\sum_{m=1}^{p} \sum_{n=1}^{q} f(m, n)}{p q}
$$

here. $f(m, n)$ is used to signify the pixels count of the pixel location $(m, n) . \mu$ is used to determine the average pixel intensity whereas, $p$ and $q$ are the intensity levels of images [4].

\subsubsection{With references evaluation metrics}

\section{1) Peak Signal to Noise Ratio (PSNR)}

PSNR is one of the evaluation metric that is used to determine the quality of an image by measuring the similarity between the reference image and resultant fused image. The higher value of PSNR means better fusion results [7]. The PSNR value is computed using equation 4 as given below:

$$
P S N R=10 \log _{10}\left\{\frac{r^{2}}{M S E}\right\}
$$

Here, $r$ indicates the peak value of the fused image. The higher PSNR value determine the less distorted image and closer resultant image to the input image. Here, MSE stands for mean squared error. It is useful to measure the average of the square of the errors. 


\section{2) Mutual information (MI)}

Mutual information provides the information quantity detail of input images, which are merged in the resultant image. The highest Mutual information represents the effectiveness of the IF technique [1]. This metric is represented as

$$
M I_{A F}=\sum_{a, f} P_{A, F}(a, f) \log \left[\frac{P_{A F}(a, f)}{P_{A}(a) P_{F}(f)}\right]
$$

Where $P_{A}(a)$ and $P_{F}(f)$ denote the marginal histogram of input image A and fused image $\mathrm{F}$. $\quad P_{A, F}(a, f)$ denotes joint histogram of input image $\mathrm{A}$ and fused image F. If MI value is high it means fusion performance is good.

\section{3) Structured similarity index (SSIM)}

SSIM quantifies the similarity between one or more images. It is designed by modeling any contrast distortion and radiometric. It is combination of the luminance image distortion and combination of contrast distortion, loss correlation and structure distortion between source images and the final image $[1,9]$. This metric is defined as follow:

$$
\operatorname{SSIM}(x, y)=\frac{\left(2 \mu_{x} \mu_{y}+c_{1}\right)\left(2 \sigma_{x y}+c_{2}\right)}{\left(\mu_{x}^{2}+\mu_{y}^{2}+c_{1}\right)\left(\sigma_{x}^{2}+\sigma_{y}^{2}+c_{2}\right)}
$$

Where $\mu_{x}$ the average of $\mathrm{x}, \mu_{y}$ the average of $\mathrm{y}, \sigma_{x}^{2}$ the variance of $\mathrm{x}, \sigma_{y}^{2}$ the variance of $\mathrm{y}, \sigma_{x y}$ the covariance of $\mathrm{x}$ and $\mathrm{y}$ and $c_{1}$ and $c_{2}$ two variables to stabilize the division with weak denominator.

\subsection{Result Analysis of the proposed technique with other image fusion techniques}

In this experiment, MRI and FDG image dataset has been taken and fusion has been performed using the proposed technique. Additionally, results of the proposed image fusion technique has been compared with the existing technique such as MSVD and PCA. PCA is used as a statistical tool employed for the transformation of correlated variables into the uncorrelated variables. It has taken the direction with the highest variance in its first component. Subsequently, it has constructed the subspace perpendicular to the previous step in the next component. Afterwards, the maximum difference components in the subspace has been taken with respect to the perpendicular components and so on [8]. Edge information is not clear in PCA. The signals are divided into two parts as low pass and high pass filters using MSVD technique. In this, some information get lost in the first level of decomposition and continued to loss on further levels of decomposition. This process of decomposition is repeated again and again till got the successive levels [4]. The resultant image obtained from MSVD is blurred and also captured the less content information. 
Whereas, the proposed technique has given the better edge content information and also overcome the drawbacks of previous techniques.

Figure 2 shows the qualitative results of the proposed work in comparison to MSVD and PCA by using the colored images. Total 50 pairs of images are used for this purpose. The experimental results have been taken on multimodal image pairs which have been publicly available online. For the purpose of comparative analysis, proposed technique has been compared with MSVD and PCA on a pair of multimodal (MRI, FDG) images. The qualitative as well as quantitative evaluation is performed for these techniques.

Visual results of diverse techniques are illustrated in Figure 2 whereas Figure 2(a) and Figure 2(b) illustrated the MRI and FDG as two input images respectively. The qualitative results of image fusion obtained by PCA and MSVD methods are shown in Figure 2(c) and Figure 2(d) respectively. Whereas, Figure 2(e) is depicted the qualitative results of image fusion by the proposed technique. From Figure 2(e), it can be observed that the better quality of visualization in terms of sharpened image has been achieved by the proposed method as it is able to capture more information from both the source images. On the contrary, MSVD has extracted the less contents information and produced the blurred image as illustrated in Figure 2(c). Moreover, from Figure 2(d), it can be clearly observed that the edge information is not clear in the resultant fused image.

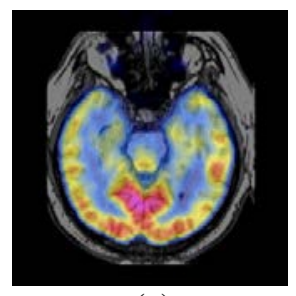

(a)

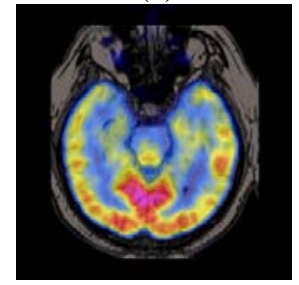

(d)

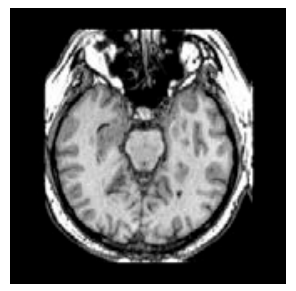

(b)

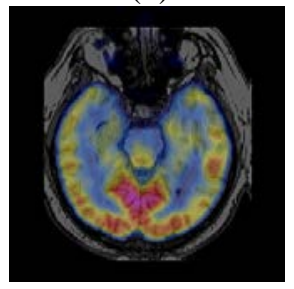

(e)

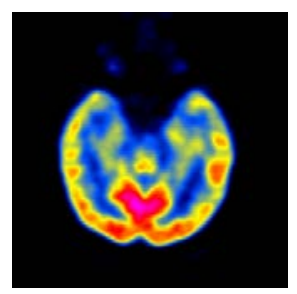

(c)

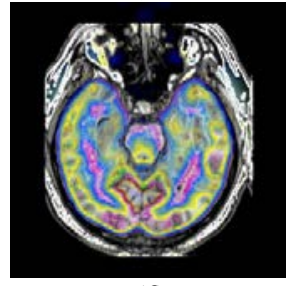

(f)

Figure 2. Visual results of multimodal images (a) Reference image (b) MRI (c) FDG (d) PCA (e) MSVD (f) Proposed technique. 
The visual results of different techniques are illustrated in Figure 3 in which Figure 3(a) and Figure 3(b) illustrated the two source images (MRI and FDG), respectively. The resultant image of fusion achieved by proposed technique is shown in Figure 3(e) whereas Figure 3(c) and Figure 3(d) illustrated the qualitative results of image fusion performed by PCA and MSVD technique, respectively.

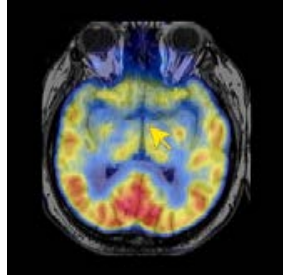

(a)

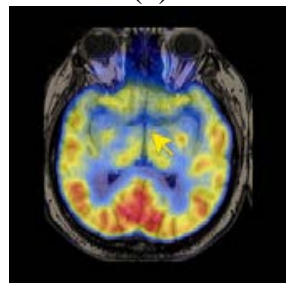

(d)

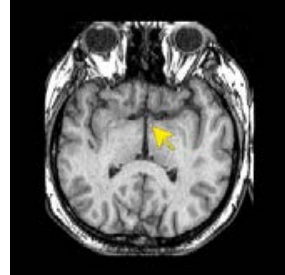

(b)

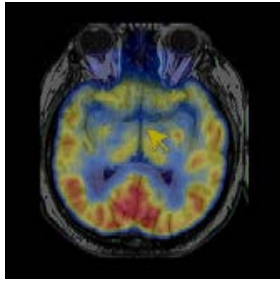

(e)

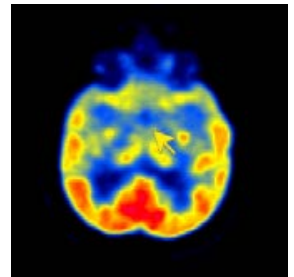

(c)

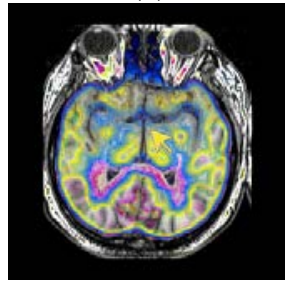

(f)

Figure 3. Visual analysis of various multi modality images by various methods (a) Reference image (b) MRI (c) FDG (d) PCA (e) MSVD (f) Proposed technique.

For the quantitative evaluation of multimodal IF techniques, various types of evaluation metrics i.e. "with reference" and "without reference" have been used which are reported in Table 1. From the table 1, it has been clearly seen that the proposed technique has obtained better quantitative results of SD, Mean, Entropy, PSNR, MI, SSIM as compared to MSVD and PCA. The proposed technique is able to restore information from both the source images. It has been observed from the quantitative and qualitative results that the proposed technique has performed well and provided much better results in comparison to other multimodal image fusion techniques.

Figure 4 illustrated the quantitative results of various techniques based on SSIM evaluation metric. The line graph depicted the results of 20 images. The proposed technique has provided the higher value of SSIM metric. This approach has obtained the approximate $1 \%$ and $2.3 \%$ SSIM metric values than the existing methods. In this technique, the structure similarity of the fused image has been displayed with respect to the reference image. 
Harpreet Kaur et al.

Table 1. Performance comparison of multi modality images for different fusion techniques

\begin{tabular}{cccc}
\hline Techniques/Metrics & SD & Entropy & PSNR \\
\hline MSVD & 0.011 & 3.991 & 29.572 \\
\hline PCA & 0.013 & 4.088 & 29.575 \\
\hline Proposed technique & 0.002 & 4.288 & 36.651 \\
\hline
\end{tabular}

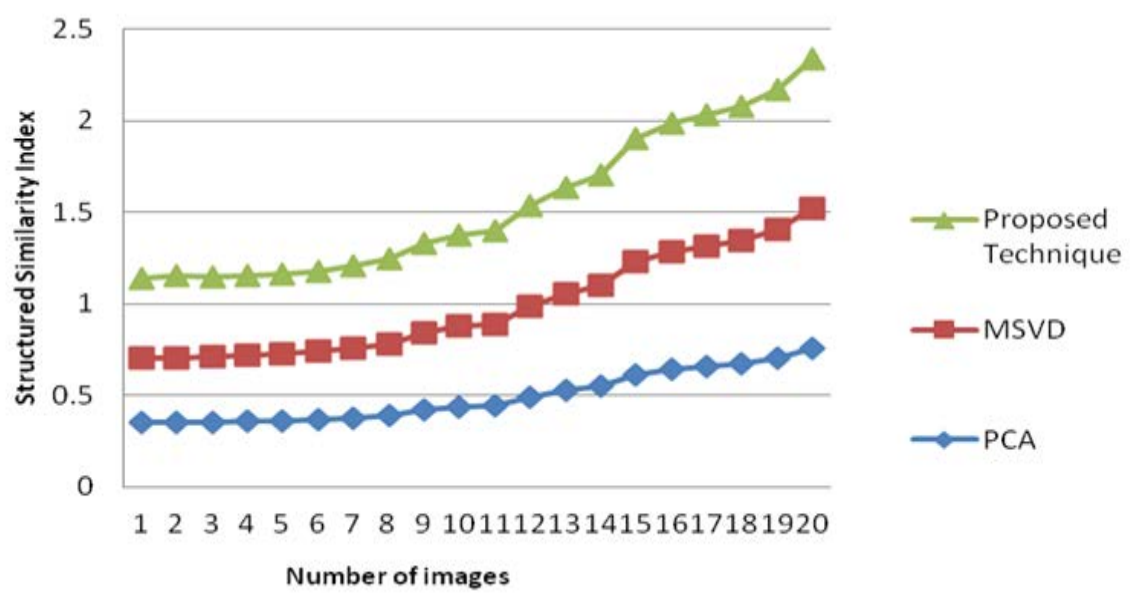

Figure 4. Quantitative evaluation of different techniques using SSIM metric.

Figure 5 and figure 6 are also illustrated the quantitative results of various techniques based on MI and mean evaluation metrics, respectively. The bar graph represented the results of 20 images. MI is used to provide the content information with respect to the source images. Figure 5 has shown the values of MI metric where fluctuations in values can be easily seen. This highest value in graph depicted that the proposed technique has provided the more content information. These metric values are lie between 1.14 and 1.2 approximately. Moreover, Mean is used to show the contrast of the image with respect to reference image. Figure 6 has shown that the mean values are decreasing with respect to the other techniques but its value is always high as compared to other techniques. These values are lie between 0.27 and 0.04 approximately. 


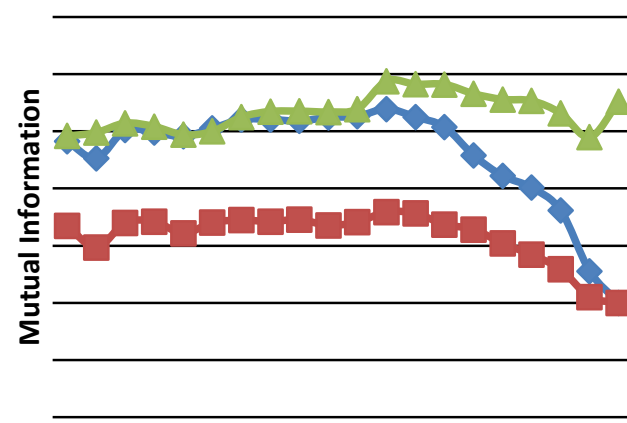

Number of images

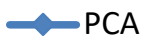

$-M S V D$

Proposed

Technique

Figure 5. Quantitative evaluation of different techniques using MI metric.

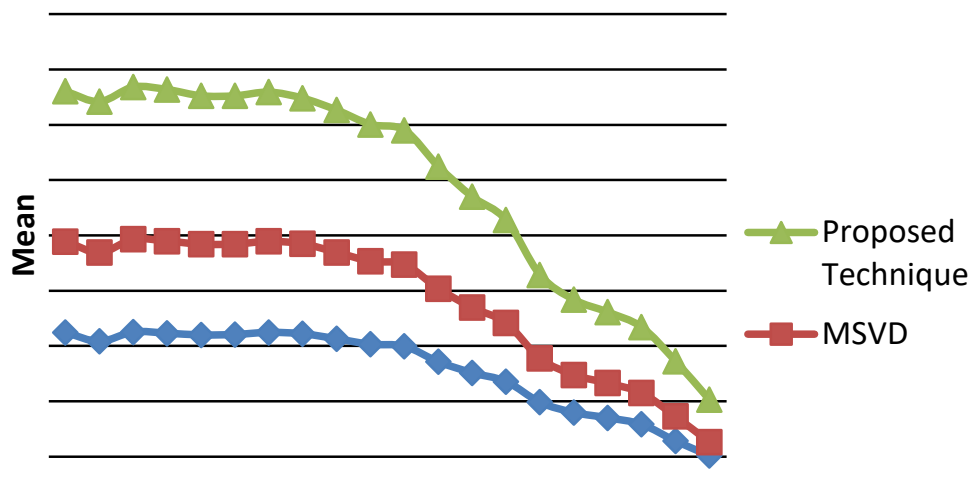

Number of images

Figure 6. Quantitative evaluation of different techniques using Mean metric.

\section{CONCLUSION}

In this paper, the information held by two or more images is collaborated into a single image which is called fused image. The technique employed to produce fused image is called image fusion. The image thus produced is more informative and it holds significant data from all the source images that participated in IF process. Traditionally, several techniques were employed in IF, but due to some limitations all of them were found less efficient. During the fusion process of two images, there is highest risk that final fused image could be corrupted in terms of noise, less efficient edges etc. Various edge preserving techniques have been developed till now in order to preserve the edges of the images but lacks to produce the qualitative output while implementation. This generates an analysis to various edge preserving IF 
Harpreet Kaur et al.

techniques and concludes that the proposed technique based on guided filters are considered to most proficient than other filters. Thus a novel edge preserving image fusion technique is developed by using the guided filters. The simulation is done by using mutli-modal datasets of medical images i.e. one is comprised of grey scale images and other is comprised of color images. The performance is evaluated in various terms i.e. MI, SSIM, Entropy, PSNR etc. On the basis of the observed facts from the results, the proposed IF technique is found to be more effective than the traditional techniques i.e. MSVD and PCA. The proposed work performs effectively to fuse the medical images whether these images are of gray scale format or color format. In future, more amendments could be done in present work, as no preprocessing techniques have been applied to the image before fusing them, therefore in future work, the image preprocessing techniques could be applied.

\section{Conflict of interest}

The authors declare that there is no conflict of interest.

\section{References}

[1] Yang Y, Zheng W, Huang S, “ Effective multifocus image fusion based on HVS and BP neural network,” The Scientific World Journal 2014 (2014) pp. 1-10.

[2] Kaur H, Rani E J, “Analytical comparison of various image fusion techniques,” International Journal of Advanced Research in Computer Science and Software Engineering 5 (2015).

[3] Garg R, Gupta P, Kaur H, “Survey on multi-focus image fusion algorithms," Recent Advances in Engineering and Computational Sciences (RAECS) (2014) pp. $1-5$.

[4] Bavirisetti DP, Kollu V, Gang X, Dhuli R. Fusion of MRI and CT images using guided image filter and image statistics. International Journal of Imaging Systems and Technology. 2017 Sep;27(3):227-37.

[5] Li, S., Kang, X., \& Hu, J. (2013). Image fusion with guided filtering. IEEE Transactions on Image Processing, 22(7), 2864-2875.

[6] Naidu VP. Image fusion technique using multi-resolution singular value decomposition. Defence Science Journal. 2011;61(5):479-84.

[7] Sireesha C, Valli B. Enhancement of Image Fusion Using Multi-Resolution Singular Value Decomposition Technique. International Journal of Latest Trends in Engineering and Technology (IJLTET). 2015 Sep 1;6 :150-158.

[8] Naidu VP, Raol JR. Pixel-level image fusion using wavelets and principal component analysis. Defence Science Journal. 2008 May 1;58(3):338. 
[9] Bai L, Xu C, Wang C. A review of fusion methods of multi-spectral image. OptikInternational Journal for Light and Electron Optics. 2015 Dec 1;126(24):4804-7.

[10] Rana A, Arora S. Comparative analysis of medical image fusion. International Journal of Computer Applications. 2013 Jan 1;73(9).

[11] Behzad Kalafje Nobariyan, Sabalan Daneshvar, Andia Foroughi, A new MRI and PET image fusion algorithm based on pulse coupled neural network.2014.ICEE.1950-1955.

[12] Ma J, Ma Y, Li C. Infrared and visible image fusion methods and applications: a survey. Information Fusion. 2019 Jan 1;45:153-78.

[13] Dogra A, Goyal B, Agrawal S. From multi-scale decomposition to non-multiscale decomposition methods: A comprehensive survey of image fusion techniques and its applications. IEEE Access. 2017;5:16040-67.

[14] Li S, Kang X, Fang L, Hu J, Yin H. Pixel-level image fusion: A survey of the state of the art. Information Fusion. 2017 Jan 1;33:100-12.

[15] Bhat, S., \& Koundal, D. (2021). Multi-focus Image Fusion using Neutrosophic based Wavelet Transform. Applied Soft Computing, 106, 107307.

[16] Bhat, S., \& Koundal, D. (2021). Multi-focus image fusion techniques: a survey. Artificial Intelligence Review, 1-53.

[17] Kaur, H., Koundal, D., \& Kadyan, V. (2021). Image fusion techniques: a survey. Archives of Computational Methods in Engineering, 1-23.

[18] Bhat, S., \& Koundal, D. (2020). Multi-focus Image Fusion: Quantitative and Qualitative Comparative Analysis. In Proceedings of ICRIC 2019 (pp. 533-542). Springer, Cham.

[19] Kaur, H., Koundal, D., \& Kadyan, V. (2019, April). Multi Modal Image Fusion: Comparative Analysis. In 2019 International Conference on Communication and Signal Processing (ICCSP) (pp. 0758-0761). IEEE.

[20] Chitkara, K. B., Sharma, B., \& Koundal, D. (2019, March). Comparative Analysis of Image Fusion Methods. In 2019 6th International Conference on Computing for Sustainable Global Development (INDIACom) (pp. 535-541). IEEE. 